\title{
Essential tips for a rock-solid geoscience PhD: Part I
}

\section{Thinking of doing a PhD? In the first of a three-part focus, Melanie Leng \& Anson Mackay explain how to get started on the right foot}

Embarking on a $\mathrm{PhD}$ is a big decision. It will consume 3 to 4 years of your life, so why do it? Well, maybe because you want to learn more about the world and wish to challenge yourself? Maybe you want to develop as a person and improve your abilities to understand and solve problems? Or maybe you want to become a better communicator, to be able to engage with a wide audience of both experts and non-experts alike. Doing a PhD should increase your confidence and time management. Plus, if you have a lifelong fascination with something in geoscience then you should be able to tailor a doctorate to suit your particular interests. Your PhD could set your future employment opportunities and influence the rest of your life. There's a lot to consider when choosing a PhD, from the discipline and project to pursue, to the university you study at.

Together, we've supervised over 100 geoscience PhD students through to graduation. Our advice is not exhaustive, but it comes from decades of personal experiences as $\mathrm{PhD}$ supervisors, as well as from published and on-line observations. There is no doubt that doing a PhD can be a stressful process, and thankfully universities are beginning to acknowledge the scale of the problem. So, take your time choosing a PhD-it's a big decision! Think in advance about your own expectations and those of the supervisor. There are many things to consider, but hopefully some of the advice below will make the experience a more enjoyable one.

\section{Choose your supervisor}

It may be a killer project, but it's important to know who your supervisors are. After securing an interview or meeting, do some research. Look up the potential supervisor(s) and their laboratory or research group via their websites, and online presence. Do they appear interesting and are they doing research that genuinely interests you? Look at how many other PhD students and postdocs there are, and take time to consider the diversity of the research group. Do they look like a dynamic and diverse research group that you would want to feel part of? You may wish to contact current and former PhD students of that supervisor to get a feel for their experiences in the group. You may also want to find out if any staff member in your undergraduate school is in the same field. If so, ask if they know anything about the research group you may be joining (but be mindful, all relationships are different and they might see themselves as competitors).

Which research group does the potential supervisor sit in within the School? Is it relevant to the project? Are there others in the School doing similar research? These may seem like minor issues, but being part of an internal research team can lead to a much richer experience.

If you get an interview, prepare some questions before hand. Ask about the project, the postgraduate environment in the School, how often supervisory meetings will be held, what training and teaching experience will be offered, what the work-life balance culture is like.

You have to spend 3 to 4 years working closely with your supervisors, so make sure you can.

\section{Student-supervisor relationships}

In the UK, geoscience PhD students typically have 2 supervisors, but you may also have several more (external/unofficial/collaborators) to cover the many different facets of your research. The first supervisor is usually key and will do most of the supervision and administration.

Ensure you have regular official meetings, at least every few weeks in the early stages. Most universities in the UK require documented meetings every month or so, and it is your responsibility to arrange these. Many universities now have electronic log books where meetings can be written up and signed off on-line. Try to get your second supervisor to attend, too. Create an agenda before the meeting, write notes in the meeting and type these up as minutes with actions (and agreed deadlines). E-mail the minutes around soon after the meetings. If relevant, upload these into an electronic log. Make sure your (evolving) career goals are regularly discussed with your supervisors.

Doing a PhD can be stressful, and personal support is needed, but keep these supervisory meetings efficient and productive, focussed on the project, especially if more than one advisor is present. Arrange separate meetings to discuss any personal issues relevant to progress of the $\mathrm{PhD}$ (these could also be with your second supervisor or postgraduate tutor). Ideally such meetings should take place before the main meeting, so that any progress and action points discussed are done so with the previous discussions in mind. Informal meetings over coffee are also beneficial.

Your supervisors are not your friends (although your relationship may develop into a close friendship, post PhD). It's probably not a good idea to "Friend" your supervisor on Facebook (or vice versa), at least until you have finished your PhD.

If at any time in your PhD you feel that the relationship with your supervisor is deteriorating, seek advice immediately from your departmental graduate tutor. All universities have clear harassment and bullying policies, often called dignity at work statements, which your departmental graduate tutor will be aware of. Harassment and bullying can take many forms, and knowing how these can manifest is important.

\section{Keeping in touch}

If you don't have the opportunity to see your supervisors for a few weeks, then send an 
Geoscientists in the news and on the move in the UK, Europe and worldwide

e-mail update. A few sentences are fine, and if you want to cover more than one topic then number the topics. As you become more independent contact time may decrease.

If you e-mail correspond with other supervisors/collaborators, it's a good idea to CC (Carbon Copy) your main supervisor too-a CC link usually means that they don't have to respond. If emailing a large number of people, use the BCC (Blind Carbon Copy) feature. Individual e-mail addresses are then invisible to recipients, preventing unwanted emails that can result from the "Reply to All" feature.

\section{Work-life balance}

Most academics consider their work as a vocation. There is a culture of working long hours, although this should not be expected. Be mindful of tight spots in the academic year-periods of exams, marking, heavy teaching weeks, application deadlines. Don't be put off by this culture; you can succeed working 9-5, 5 days a week, but you will need to be flexible when deadlines loom. Many geoscience $\mathrm{PhD}$ projects involve fieldwork, which involve longer hours. Bear in mind that coffee/tea breaks are useful for building relationship, as well as discussing approaches and ideas. Time away from your desk can be enlightening. Have regular breaks, but keep them to a schedule.

Taking work home should not be the norm, but deadlines may require it. Sometimes taking a difficult paper or data set home to look at in the quiet and comfort of your sofa can be revelational. Try to be efficient, aim to achieve something every day, even if the accomplishment is small (and write these down to discuss at your supervisory meetings).

Have a life outside of your PhD. Get out at weekends, spend time with your friends and family, participate in a sport or hobby.

People who only work, potentially either burn out or are more likely to develop mental health issues.

The mental health of $\mathrm{PhD}$ students is precarious: postgraduate students are up to six times more likely to experience depression and anxiety compared to the general population. Your mental (and physical) health is extremely important. Universities offer welfare services, well-being clinics and stress management courses, and you can also seek help through your GP (remember to register with your local practise). It is important to get plenty of sleep, learn how to shut off in the evenings and weekends.

Remember, there are those around you who are going/have gone through similar experiences. Peer support is a vital (although not often spoken about) form of support while doing a $\mathrm{PhD}$, so try and put into place a good support network. Attend School social events, especially those organised for $\mathrm{PhD}$ students. And talk to your supervisors, they may be more understanding than you think.

Look out for parts II and III, which include advice on data management, giving presentations, writing up your thesis and planning for your career beyond the PhD, in the October and November issues of Geoscientist.

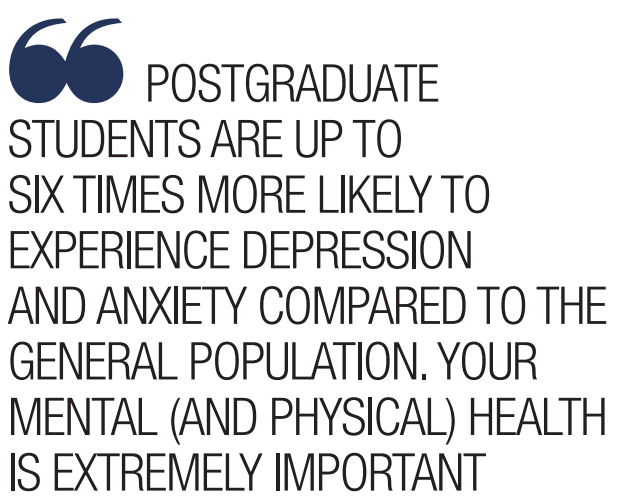

Melanie Leng ${ }^{1}$ is Director of Geochemistry at the British Geological Survey, UK, and Professor in Isotope Geoscience at the University of Nottingham, UK.

Anson Mackay ${ }^{2}$ is Professor in Environmental Change at UCL, UK, and an Honorary Research Associate at the British Geological Survey.

1mjj@bgs.ac.uk

2ans.mackay@ucl.ac.uk

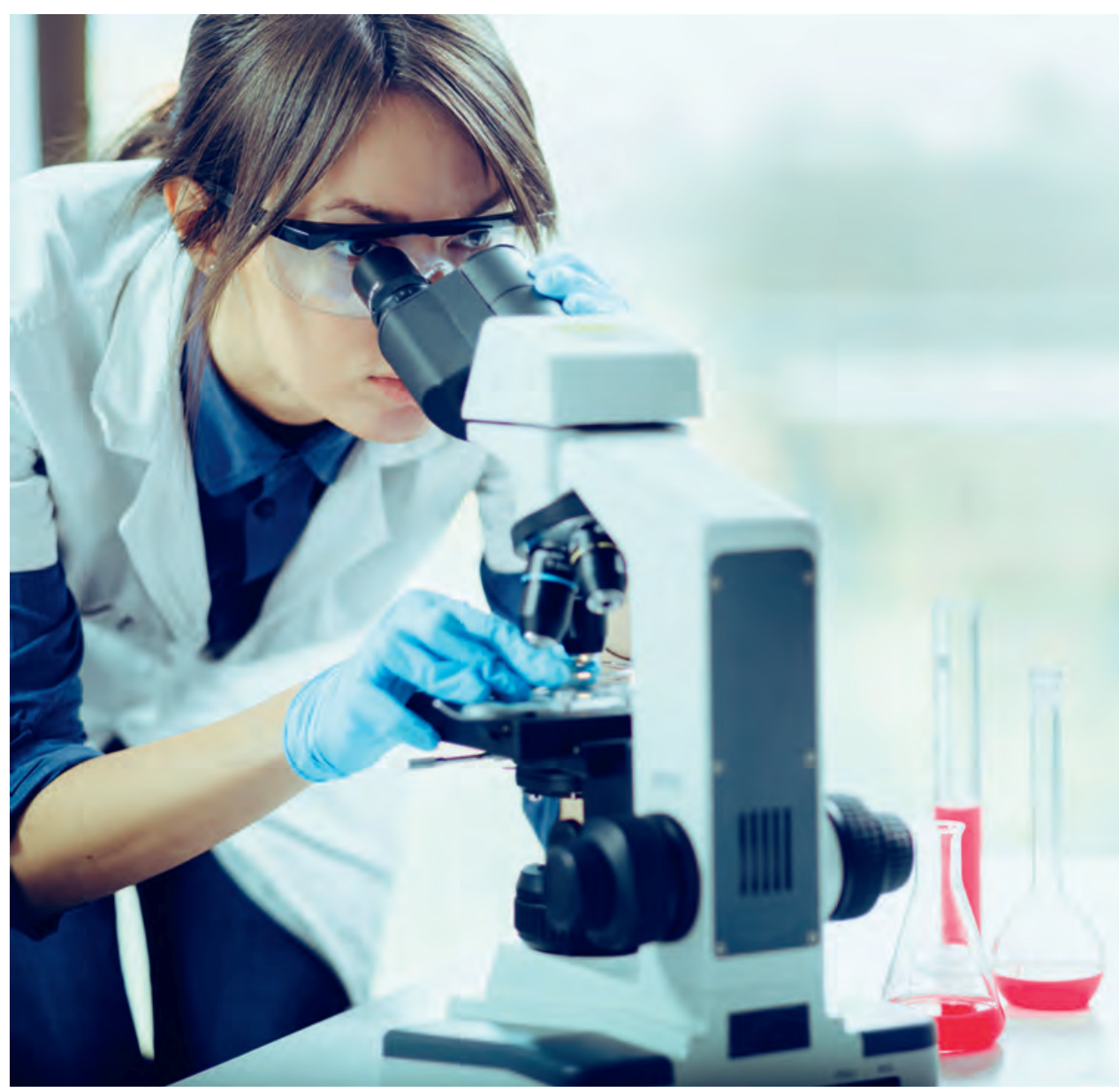

